\title{
Select Optimal Chemical Detection Equipments Using Fuzzy Decision Making Method
}

\author{
Ying Bai \\ Dept of Computer Science and Engineering ,Johnson C. Smith University, North \\ Carolina, USA \\ (ybai@jcsu.edu) \\ Dali Wang \\ Dept of Physical, Computer Science and Eng, Christopher Newport University, \\ Virginia, USA \\ (dwang@pcs. cnu .edu)
}

\begin{abstract}
:
The purpose of this research is to develop a universal model with a practical system to evaluate, identify and select an optimal system or device to perform the desired task from a large collection of available systems that have multiple objectives based on a fuzzy multiple criteria decision making model (FMCDMM). As an example, here we are using this research to identify and select an optimal detection system or device to detect hazardous chemical materials.
\end{abstract}

Keywords: Fuzzy multi-criteria decision making, optimal evaluations, explosive detection equipments.

\section{INTRODUCTION}

Continuously check and detect high-threat chemical materials to provide early warning and control are critical parts in protecting chemical threats to our territory.

Chemical detection equipment (CDE) is an essential component of hazardous material (HAZMAT) emergency response. It can be found that the main challenge with these detection technologies is to select and identify the best equipment from a large of collections of available detection devices based on a quite few of factors or criteria. Dena et al [2] reported a review for over 24,000 citations and identified 55 detection systems and 23 diagnostic decision support systems. Only 35 systems have been evaluated: 4 reported both sensitivity and specificity, 13 were compared to a reference standard, and 31 were evaluated for their timeliness. Most evaluations of detection systems and some evaluations of diagnostic systems for bioterrorism responses are critically deficient. Because false-positive and false-negative rates are unknown for most systems, decision making on the basis of these systems is seriously compromised.

Multiple criteria decision making (MCDM) was introduced as a promising and important field of study in the early 1970'es. Since then the number of contributions to theories and models, which could be used as a basis for more systematic and rational decision making with multiple criteria, has continued to grow at a steady rate. A number of surveys, cf e.g. Bana e Costa [3], show the vitality of the field and the multitude of methods which have been developed. When Bellman and Zadeh, and a few years later Zimmermann, introduced fuzzy 
sets into the field, they cleared the way for a new family of methods to deal with problems which had been inaccessible to and unsolvable with standard MCDM techniques.

There are many variations on the theme MCDM depending upon the theoretical basis used for the modeling. Zeleny [4] shows that multiple criteria include both multiple attributes and multiple objectives, and there are two major theoretical approaches built around multiple attribute utility theory (MAUT) and multiple objective linear programming (MOLP), which have served as basis for a number of theoretical variations. Bana e Costa and Vincke [5] argue that with MCDM the first contributions to a truly scientific approach to decision making were made, but find fault with the objectives to carry this all the way as we have to deal with human decision makers who can never reach the degree of consistency needed.

When fuzzy set theory was introduced into MCDM research the methods were basically developed along the same lines. There are a number of very good surveys of fuzzy MCDM (cf [6-8] and Ribeiro's contribution in this issue), which is why we will not go into details here but just point to some essential contributions. One of the good surveys is done by Chen and Hwang [9]: they make distinctions between fuzzy ranking methods and fuzzy multiple attribute decision making methods, which contain all the families (i)- (iv) listed above.

Cheng and Mon [10] propose a new algorithm for evaluating weapon systems by the Analytical Hierarchy Process (AHP) based on fuzzy scales. The systematic procedures used by Saaty's AHP method [11] results in a cardinal order, which can be used to select or rank alternatives. Cheng and Mon derive a simple and general algorithm for fuzzy AHP by using triangular fuzzy numbers, $\alpha$-cuts and interval arithmetic. Triangular fuzzy numbers $\sim 1$ to $\sim 9$ are used to build a judgment matrix through pair-wise comparison techniques. They estimate the fuzzy eigenvectors of the judgment matrix by using an "index of optimism", indicating the degree of satisfaction of the decision maker. The proposed technique is illustrated with the selection of an anti-aircraft artillery system from several alternatives.

In this study, we will design and implement a FMCDM algorithm with a real system to improve the evaluation and selection process of the optimal detection devices for the CAs to enable users to identify and select the optimal CAs devices from a large of collections of candidates in more accurate and convenient ways.

An introduction to this study and a technique review of MCDM are given in section 1 . The detailed description of the FMCDM model is described in section 2. The proposed FMCDM algorithm used to estimate the weight to the associated criteria or objectives is provided in section 3. A case study with an example of evaluating and assessing the optimal CDE is given in section 4. Section 5 provides the conclusion.

\section{FUZZY MCDM MODEL}

Normally, a triangular fuzzy number $A$ can be defined by a triplet $(a, b, c)$ shown in Fig.1a. An example of a fuzzy member of an equipment cost is shown in Fig.1b. The membership function is defined as Eq. (1).

The general multi-attribute decision making (MADM) model can be described as [12]:

(a) Let $X=\left\{X_{i} \mid i=1, \ldots, m\right\}$ denote a finite discrete set of $m(\geq 2)$ possible alternatives;

(b) Let $A=\left\{A_{j} \mid j=1, \ldots, n\right\}$ denote a finite set of $n(\geq 2)$ criteria according to which the desirability of an alternative is to be judged,

(c) Let $\omega=\left(\omega_{1}, \omega_{2}, \ldots, \omega_{n}\right)^{\mathrm{T}}$ be the vector of weights, where $\sum_{j=1}^{n} \omega_{j}=1, \omega_{\mathrm{j}} \geq 0, j=1, \ldots, n$, and $\omega_{\mathrm{j}}$ denotes the weight of criterion $A_{j}$, 
(d)Let $R=\left(r_{i j}\right)_{m \times n}$ denote the $m \times n$ decision matrix, where $r_{i j}(\geq 0)$ is the performance rating of alternative $X_{i}$ with respect to criterion $A_{j}$.

$$
\mu_{A}(x)= \begin{cases}0, & x<a, \\ \frac{x-a}{b-a}, & a \leq x \leq b, \\ \frac{c-x}{c-b}, & b \leq x \leq c, \\ 0, & x>c .\end{cases}
$$

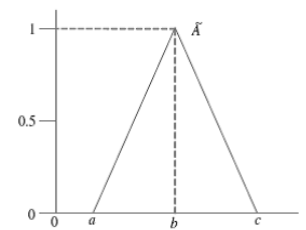

(e)

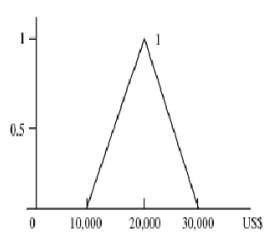

(b)

Fig.1. A representation of a triangular fuzzy member

\section{Step 1 Representation of Fuzzy Requirement}

When evaluating and assessing the optimal CDE from a number of similar alternatives, a decision maker normally develops in his/her mind some sort of ambiguity. Representation of fuzzy requirements has been introduced in the beginning of this section.

\section{Step 2 Similarity Measure}

In step 1, the requirements of selecting optimal CDE have been described as the triangular fuzzy number with respect to different criteria. In this step, we will take the requirement vector as the ideal CDE, with the purpose to measure the similarity degree with the existing candidate CDE vectors, in which the specification values are known and determinate. As we know, a fuzzy number cannot be compared with a crisp one directly unless a non-fuzzy number has to be transformed into the form of fuzzy number firstly. For example, for a crisp number $b$, the form of its triangular fuzzy can be written as the Eq. (2).

$$
b=\left(b^{L}, b^{M}, b^{U}\right)
$$

where $b^{L}=b^{M}=b^{U}$, and Similarity measure between two triangular fuzzy numbers can be calculated with Eq. (3) [13].

$$
s(a, b)=\frac{a^{L} b^{L}+a^{M} b^{M}+a^{U} b^{U}}{\max \left[\left(a^{L}\right)^{2}+\left(a^{M}\right)^{2}+\left(a^{U}\right)^{2},\left(b^{L}\right)^{2}+\left(b^{M}\right)^{2}+\left(b^{U}\right)^{2}\right]}
$$

where two triangular fuzzy numbers are $a=\left(a^{L}, a^{M}, a^{U}\right)$ that represents the ideal number and $b$ $=\left(b^{L}, b^{M}, b^{U}\right)$ that represents the real number, respectively.

\section{Step 3 Construction of Decision Matrix}

Calculation result of similarity measure between alternate CDEs and the ideal CDE can be concisely expressed in a matrix format, which is called a decision matrix in MCDM problems, and in which columns indicate CDE criteria and rows alternate CDEs. Thus, an element $s_{i j}$ in the in Eq. (4) denotes the similarity degree to the ideal CDE of the ith CDE with respect to the $j$ th criterion. 


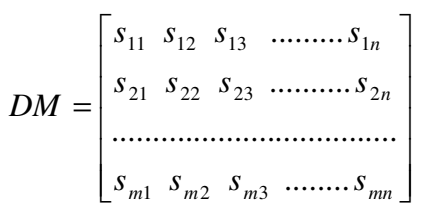

\section{Step 4 Normalization}

In order to eliminate the difference of dimension among different criteria, the operation of normalization is needed to transform various criteria dimensions into the non-dimensional criteria. Eqs. (5) and (6) are utilized to perform this normalization [12]

$$
\begin{gathered}
r_{i}=\left(\frac{a_{i}}{c_{i}^{\max }}, \frac{b_{i}}{b_{i}^{\max }}, \frac{c_{i}}{a_{i}^{\max }} \wedge 1\right) \text { for benefit criteria } \\
r_{i}=\left(\frac{a_{i}^{\min }}{c_{i}}, \frac{b_{i}^{\min }}{b_{i}}, \frac{c_{i}^{\min }}{a_{i}} \wedge 1\right) \text { for cost criteria }
\end{gathered}
$$

where $(\bullet)^{\max }{ }_{\mathrm{i}}=\max \left\{(\bullet)_{\mathrm{i}}\right\}$ and $(\bullet)^{\min }=\min \left\{(\bullet)_{\mathrm{i}}\right\}$.

\section{Step 5 Rank of the Alternate Products}

The element $s_{i j}$ in the decision matrix reflects the closeness degree of the ideal CDE with the ith alternate $\mathrm{CDE}$ with respect to the $j$ th criterion. In this step, we can use the simple additive weighting (SAW) method, which is widely used in MCDM, to calculate the relative importance value with respect to all criteria, with which the ranking order of alternate CDEs according to the relative importance value can be obtained. And we can consider the CDE with the highest relative importance value as the closest one to that of the decision maker requires. The relative importance value of ith alternate CDE can be calculated with Eq. (7).

$$
U_{i}=\sum_{j=1}^{n} x_{i j} \omega_{j} \quad i=1,2, \ldots, m
$$

And the maximum of relative importance value can be written as Eq. (8).

$$
U_{\text {nax }}=\max _{\mathrm{i}} \sum_{j=1}^{n} x_{1 j} \omega_{j} \quad i=1,2, \ldots, m
$$

\section{ALGORITHM OF WEIGHT ASSIGNMENT}

Compared with most MCDM methods, the FMCDM model reported by Bin Zhu et al. is one of the simplest and most effective methods [12]. A similarity measure method is utilized to build the decision matrix. However, one problem of this method is that the weight associated with each alternative is estimated or determined by the decision maker or an evaluation team based on their experience. Generally, this kind of weight estimation is acceptable for a small set of alternatives, such as 5 or less than 10. However, for a large set of alternatives, which is a popular situation, this weight estimation is not accurate and correct based only on the decision make's experience.

There are some different weight estimation methods reported by researchers, such as Saaty who developed a paired comparison matrix and then an eigenvector that is equivalent to the weight for an associated alternative can be calculated based on that paired comparison matrix 
[14]. Yager multiplied the normalized eigenvector by the order of the system to obtain exponents for weighting the fuzzy criteria in a decision problem [15]. However, both methods need a lot of mathematical operations and therefore make the process very complicated and time consuming.

In this paper, we adopted a weight estimation method based on the paired comparison matrix to simplify this estimation process. The operational procedure is completed by the following steps:

1. List the degree of importance or importance level of each criterion relative to another criterion based on Table 1 [16].

2. Construct a paired comparison matrix $\mathrm{W}$ based on the importance levels in step 1. Each element $\mathrm{w}_{i j}$ in the matrix $\mathrm{W}$ is a ratio of the importance level of the ith criterion over to the jth criterion.

3. Add each row $\omega_{i}=\sum_{j=1}^{n} \omega_{i j} ;$ where $i=1 \sim \mathrm{n}$.

4. Calculate the normalized weight factor for each criterion $\omega_{n i}=\frac{\omega_{i}}{\omega_{n}} ;$ where $\omega_{n}=\sum_{i=1}^{n} \omega_{i}$.

5. These weight factors can be used to build the decision matrix based on the similarity measure method.

Table 1: Weight assignment to a paired comparison

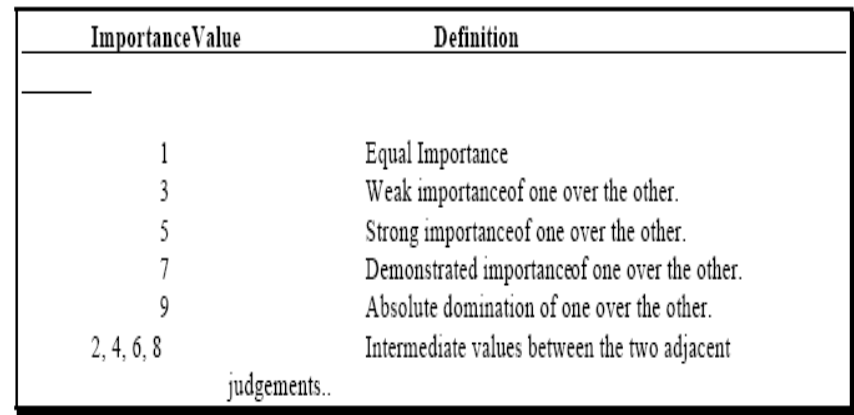

In the following section, we will use an example to illustrate this weight estimation method.

\section{CASE STUDY}

In this section, we use a group of CDEs [17] that is collected by the National Institute of Standards and Technology (NIST) and the National Homeland Security Research Center (NHSRC), which contains the updated evaluation results for all equipments used to detect CAs and Toxic Industrial Chemicals/Toxic Industrial Materials (TICs/TIMs), as an example to illustrate the weight estimation method we developed in this proposal with the Fuzzy MCDM model we discussed in section 3 to evaluate and select the optimal CDE currently used in the U.S. Homeland Security system.

According to [18], the CDEs can be divided into seven (7) categories based on the usage:

1. Handheld-portable detection equipment

2. Handheld-stationary detection equipment

3. Vehicle-mounted detection equipment

4. Fixed-site detection systems 
5. $\quad$ Fixed-site analytical laboratory systems

6. Standoff detection systems

7. Detection systems with limited data

For each category, the CDEs can be further grouped into three sub-categories: the CDEs that capable of detecting CAs only, the CDEs that capable of detecting TICs/TIMs only, and the CDEs that capable of detecting both CAs and TICs/TIMs. To make our research simple and easy to be understood, in this study, we will concentrate on the handheld-portable chemical detectors that capable of detecting CAs only.

Refer to 16 selection factors or criteria used to evaluate all kinds of CDEs in section 5 in [17], it can be found that one of the most important properties is that the evaluation factors are not given by crisp or accurate values, instead they are categorized and provided by different ranges. An example of the first criterion (unit cost of each CDE) is shown in Fig.2.

\begin{tabular}{|c|l|}
\hline \multicolumn{2}{|c|}{ Unit Cost } \\
\hline 0 & Less than \$500 per unit \\
\hline 0 & Between \$500 and \$2K per unit \\
\hline 0 & Between \$2K and \$5K per unit \\
\hline$\bigcirc$ & More than \$5K per unit \\
\hline
\end{tabular}

Fig. 2 The criteria for evaluating the unit cost

This kind of criterion did not provide very accurate or crisp evaluation values, but it does provide some vague or ambiguous values. Just because of these vague or ambiguous evaluation values, it is very suitable to be assessed and analyzed by using a FMCDM system, and that is the objective and key points of this proposal.

Because we are using a triangular shape as a fuzzy member (Fig.1) in this study, therefore we need to perform a little modification to all 16 criteria used for this optimal selection process.

Let us create a evaluation table for those handheld-portable chemical detectors based on the modified fifteen (15) criteria (detect CAs only, no $3^{\text {rd }}$ criterion), which is shown in Table 2 . The blank evaluation results in Table 2 indicate that no information available for that criterion for the selected CDEs. In order to utilize FMCDM system to evaluate and select the optimal handheld-portable chemical detectors that capable of detecting CAs only, we need to perform the following fuzzification operations to all criteria listed in Table 2.

First let's use the following letters to represent all 15 criteria as (the number following each criterion indicates the relative important level of that criterion):

- $\mathrm{C}_{1}$ : Unit Cost

- $\mathrm{C}_{2}$ : CAs Detected

- $\mathrm{C}_{3}$ : Sensitivity

- $\mathrm{C}_{4}$ : Resistance to Interference

- $\mathrm{C}_{5}$ : Response Time

- $\mathrm{C}_{6}$ : Start-Up Time

- $\mathrm{C}_{7}$ : Detection States

- $\mathrm{C}_{8}$ : Alarm Capability 
- $\mathrm{C}_{9}$ : Portability

- $\mathrm{C}_{10}$ : Battery Needs

- $\mathrm{C}_{11}$ : Power Capability

- $\mathrm{C}_{12}$ : Operational Environment

- $\mathrm{C}_{13}$ : Durability

- $\mathrm{C}_{14}$ : Operator Skill Level

- $\mathrm{C}_{15}$ : Training Requirements
(9)

Next we need to use the following three fuzzy levels to represent those three graphical levels shown in Table 2:

- $\mathrm{HIGH}$

- 0 : MID

- O : LOW

Table 2: Handheld-Portable Detection Equipment (CAs)

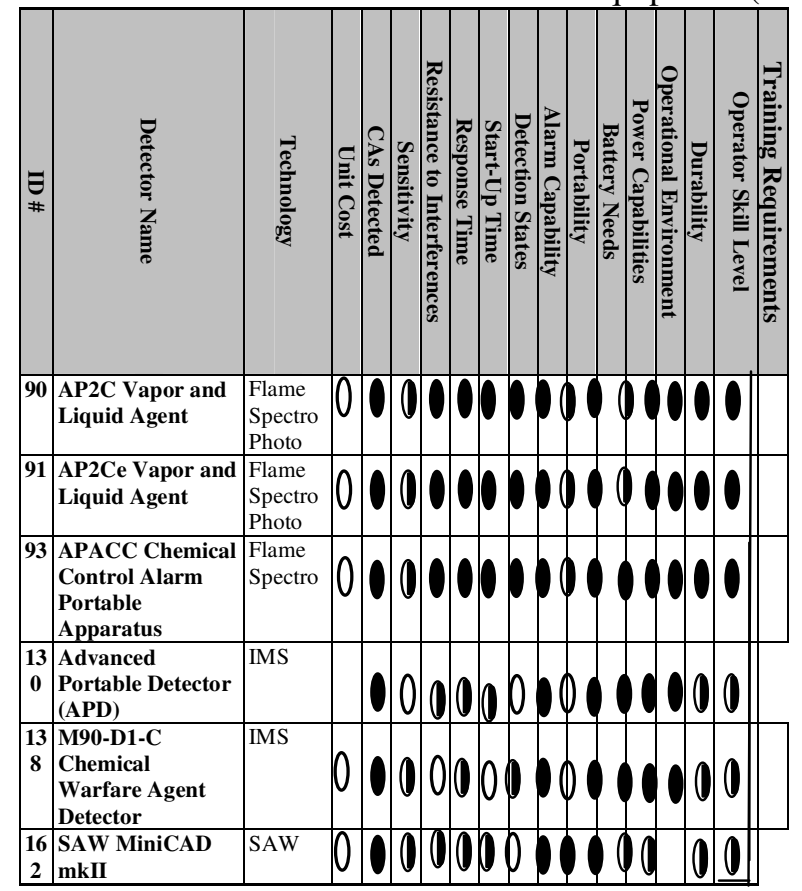

The evaluation results for six (6) handheld-portable chemical detectors that capable of detecting CAs only is shown in Table 3 when using those new definitions described above.

Also those fuzzy levels can be represented by three numbers, which are defined as:

\section{HIGH - 4, MID - 2 and LOW - 1}

Now we need to build the relative important levels for all criteria. This level is represented by a ratio between two or a paired of criteria. The ratio between the same criteria is 1 and a number represented in the intersection of a row and a column represnets the importance ratio between that row and that column. For example, the number in the intersection cell of row $\mathrm{C}_{2}$ and column $\mathrm{C}_{3}$ is 2 , which means that the criterion $\mathrm{C}_{2}$ (CAs Detected) is 2 times more important than that of criterion $\mathrm{C}_{3}$ (Sensitivity). This relative important levels is obtained based on the experience and real knowledge of evaluators or decision makers. A completed 
importance level of this example based on Eq. (9) is calculated by comparing two criteria and based on the following equations:

The important level in cell

$$
A_{i j}=\frac{\operatorname{Im} \text { por } \tan t \text { Level of } C_{j}}{\operatorname{Im} \text { por } \tan t \text { Level of } C_{i}}
$$

Table 3: Evaluation Results (CAs)

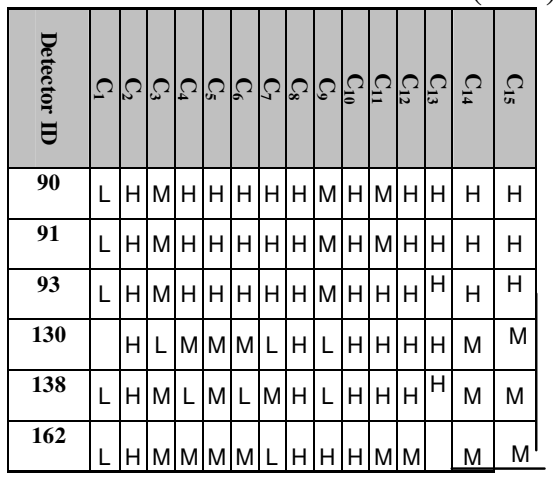

Next let's calculate the overall weight for each row by adding each row $\omega_{i}=\sum_{j=1}^{n} \omega_{i j}$; where $\mathrm{i}=$ $1 \sim \mathrm{n}$ and $\mathrm{n}=15$ is the total number of the criteria. The result of this calculation is shown in Table 4.

Table 4: The Overall Weight for Each Criterion

\begin{tabular}{|c|ccc|r|r|rr|}
\hline & $\mathbf{c 1}$ & $\mathbf{c 2}$ & $\mathbf{c 3}$ & $\mathbf{c 4}$ & $\mathbf{c 5}$ & $\mathbf{c 6}$ & $\mathbf{c 7}$ \\
\hline$\omega_{i}$ & 12.03 & 120 & 60 & 39.50 & 24.10 & 17.11 & 20 \\
\hline $\mathbf{c 8}$ & $\mathbf{c 9}$ & $\mathbf{c 1 0}$ & $\mathbf{c 1 1}$ & $\mathbf{C 1 2}$ & $\mathbf{C 1 3}$ & $\mathbf{C 1 4}$ & $\mathbf{C 1 5}$ \\
\hline 9.23 & 12.34 & 10.99 & 10 & 30 & 15.02 & 8.55 & 8.00 \\
\hline
\end{tabular}

The total weight is obtained by adding all weights of criteria together, which is 396.87 . The normalized weight is shown in Table 5.

Table 5: The Normalized Weight for Each Criterion

\begin{tabular}{|c|c|c|c|c|c|c|c|}
\hline & $\mathbf{c 1}$ & $\mathbf{c 2}$ & $\mathbf{c 3}$ & $\mathbf{c 4}$ & $\mathbf{c 5}$ & $\mathbf{c 6}$ & $\mathbf{c 7}$ \\
\hline$\omega_{i}$ & 0.03 & 0.30 & 0.15 & 0.10 & 0.06 & 0.04 & 0.05 \\
\hline $\mathbf{c 8}$ & $\mathbf{c 9}$ & $\mathbf{c 1 0}$ & $\mathbf{c 1 1}$ & $\mathbf{C 1 2}$ & $\mathbf{C 1 3}$ & $\mathbf{C 1 4}$ & $\mathbf{C 1 5}$ \\
\hline 0.02 & 0.03 & 0.027 & 0.025 & 0.076 & 0.038 & 0.02 & 0.02 \\
\hline
\end{tabular}

Now we can perform step 4 in section 3 to add these normalized weight for each criterion with all criteria to construct the ideal and criteria weight table, which is shown in Table 6.

The vector of the ideal CDEs can be represented as the following form of the triangualr fuzzy number (Table 6):

$$
\begin{aligned}
\mathrm{T}= & {[(148),(148),(148),(148),(148),(148),} \\
& (148),(148),(148),(148),(148),(148), \\
& (148),(148),(148)] ;
\end{aligned}
$$


Table 6: The Ideal CDEs and Criteria Weight

\begin{tabular}{|c|c|c|c|c|}
\hline 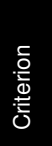 & $\begin{array}{l}\overline{\bar{J}} \\
\underline{\underline{\delta}}\end{array}$ & 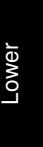 & $\begin{array}{l}\frac{\bar{d}}{0} \\
\text { 음 }\end{array}$ & 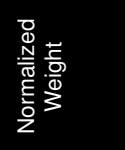 \\
\hline c1 & 4 & 1 & 8 & 0.030 \\
\hline c2 & $\overline{4}$ & $\overline{1}$ & $\overline{8}$ & 0.300 \\
\hline c3 & 4 & 1 & 8 & 0.150 \\
\hline c4 & 4 & 1 & 8 & 0.100 \\
\hline c5 & $\overline{4}$ & 1 & 8 & 0.060 \\
\hline c6 & 4 & 1 & 8 & 0.040 \\
\hline c7 & $\overline{4}$ & $\overline{1}$ & 8 & 0.050 \\
\hline$c 8$ & $\overline{4}$ & 1 & 8 & 0.020 \\
\hline c9 & 4 & $\overline{1}$ & 8 & 0.030 \\
\hline c10 & $\overline{4}$ & 1 & 8 & 0.027 \\
\hline c11 & $\overline{4}$ & 1 & 8 & 0.025 \\
\hline c12 & $\overline{4}$ & $\overline{1}$ & 8 & 0.076 \\
\hline c13 & 4 & 1 & 8 & 0.038 \\
\hline c14 & 4 & 1 & 8 & 0.020 \\
\hline c15 & 4 & 1 & 8 & 0.020 \\
\hline
\end{tabular}

The corresponding vector of the criteria weight can be expressed as:

$$
\begin{array}{r}
\omega=(0.03,0.30,0.15,0.10,0.06,0.04,0.05,0.02, \\
0.03,0.027,0.025,0.076,0.038,0.02,0.02) ;
\end{array}
$$

The corresponding vector of the criteria weight can be expressed as:

$$
\begin{array}{r}
\omega=(0.03,0.30,0.15,0.10,0.06,0.04,0.05,0.02, \\
\quad 0.03,0.027,0.025,0.076,0.038,0.02,0.02) ;
\end{array}
$$

The decision matrix, which can be calculated based on the similarity degree with respect to each criterion between the ideal CDE and the alternatives, is shown in Table 7 by using Eqs (3) - (6). The relative importance value of ith alternate CDE with respect to all criteria can be computed by equation (7), and the final calculating results are shown in Table 8 .

Table 8: The Important Level of Each Alternative CDE

\begin{tabular}{rr|l||l||l|cr|} 
CDE ID\# & $\mathbf{9 0}$ & $\mathbf{9 1}$ & $\mathbf{9 3}$ & $\mathbf{1 3 0}$ & $\mathbf{1 3 8}$ & $\mathbf{1 6 2}$ \\
& 0.5027 & 0.5027 & 0.5107 & 0.3887 & 0.4032 & 0.3996 \\
\hline
\end{tabular}

When calculate the decision matrix, the ideal CDE vector is [LOW MID HIGH] $=\left[\begin{array}{lll}1 & 4 & 8\end{array}\right]$ and the real CDE vector is selected as [LOW MID HIGH] $=\left[\begin{array}{ll}1 & 2\end{array}\right]$, respectively. The ideal criterion in Table 6, which is 3 , is equal to the HIGH in the real criterion in Table 2 for this selection.

Table 7: Decision Matrix

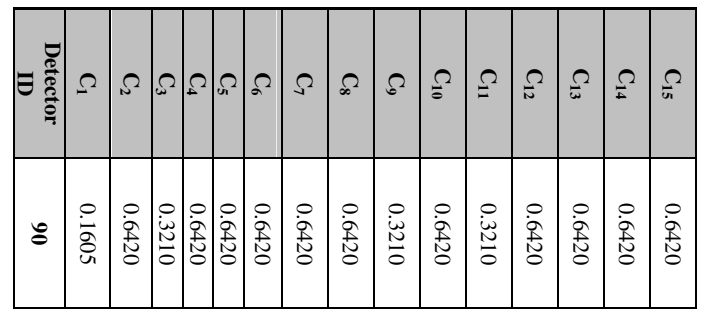




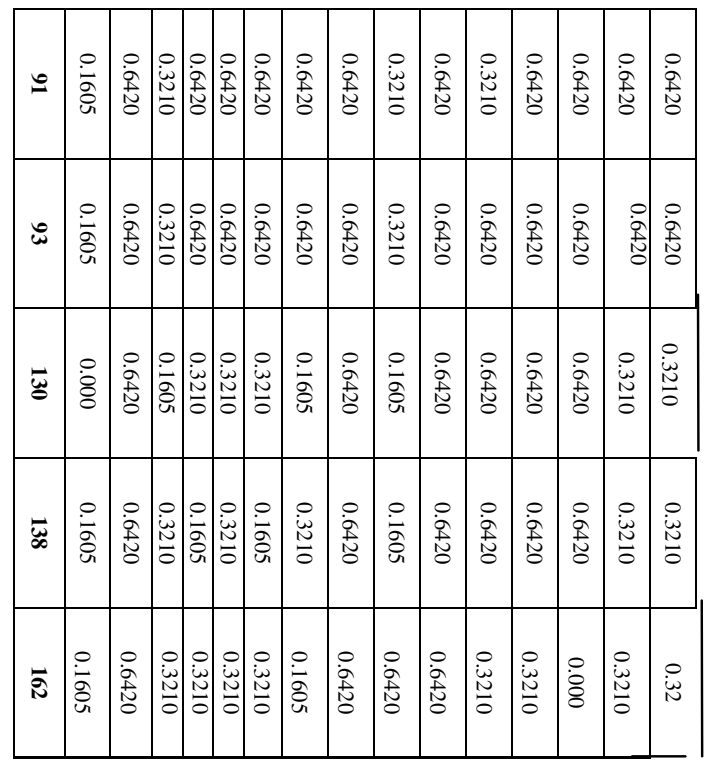

The relative importance level of $i$ th alternate CDE can be calculated with equation (7) as:

$$
U_{i}=\sum_{j=1}^{n} s_{i j} \omega_{j} \mathrm{i}=1,2, \ldots \mathrm{m} \text { ( } \mathrm{m} \text { is the number of total alternatives) }
$$

The maximum important level of CDEs can be obtained as:

$$
U_{\max }=\max _{i} \sum_{j=1}^{n} s_{i j} \omega_{j}
$$

$\mathrm{i}=1,2, \ldots \mathrm{m}$ ( $\mathrm{m}$ is the number of total alternatives)

The CDE that has the maximum important level can be found from Table 8, which is the APACC Chemical Control Alarm Portable Apparatus (Detector ID\#: 93) with the most important level of $\mathbf{0 . 5 1 0 7}$.

The results obtained from this research are based on the CDE data collected by the NIST and NHSRC in 2007. More updated evaluations can be achieved by using updated information on CDEs in the future. However, so far no any new evaluations have been released by NIST and NHSRC.

In order to properly use this Fuzzy Decision Making (FDM) method to select and identify the optimal chemical detection equipments, the following study or training requirements are necessary:

- Basic understanding about the FDM method.

- An expert group is necessary to select and define the criteria for chemical detection equipments to be selected.

- A software tool such as MATLAB is recommended to be used to perform the detailed calculations.

\section{CONCLUSIONS}

Decision making is one of the most important and popular research and application topics in the national defense and homeland security. To assess and evaluate the optimal Chemical Detecting Equipment (CDE) system from a large group of alternatives that contain multiple criteria is a 
challenging, sometimes may be a headach task to decision makers in the defense and the homeland security system. To correctly pridict and effectively protect our nationa's safety, accurately evaluate and correctly assess the optimal CDE system is the prerequsite and critical task. In this paper, we used a collection of CDE information reported by different agencies in recent years with a target example to illustrate how to use FMCDM model to simplify this assessing process.

\section{ACKNOWLEDGEMENTS}

The data used in this paper are originally published by the National Institute of Justice, U.S. Department of Justice.

\section{REFERENCES}

1) http://www.emedicine.com/emerg/TOPIC924.HTM.

2) Dena M. Bravata, Vandana Sundaram, Kathryn M. McDonald, Wendy M. Smith, Herbert Szeto, Mark D. Schleinitz, and Douglas K. Owens, Emerging Infectious Diseases. 2004, www.cdc.gov/eid Vol. 10, No. 1, January.

3) C.A. Bana e Costa and P. Vincke, 1990, Multiple Criteria Decision Aid: An Overview in C.A. Bana e Costa ed., Readings in Multiple Criteria Decision Aid, Springer Verlag, Berlin-Heidelberg, pp 314.

4) M. Zeleny, 1982, Multiple Criteria decision-making, McGraw-Hill, New-York.

5) C.A. Bana e Costa ed., 1990, Readings in Multiple Criteria Decision Aid, Springer Verlag, BerlinHeidelberg.

6) S.J.Chen and C.L.Hwang, 1993, Fuzzy Multiple Attribute decision-making, Methods and Applications, Lecture Notes in Economics and Mathematical Systems, Vol. 375, Springer, Heildelberg.

7) J.C.Fodor and M.Roubens, 1994, Fuzzy Preference Modelling and Multicriteria Decision Support, Kluwer, Dordrecht.

8) M. Sakawa, 1993, Fuzzy Sets and Interactive Multiobjective Optimization, Applied Information Technology, Plenum Press, New York.

9) S.J.Chen and C.L.Hwang, 1993, Fuzzy Multiple Attribute decision-making, Methods and Applications, Lecture Notes in Economics and Mathematical Systems, Vol. 375, Springer, Heildelberg.

10) C.H.Cheng and D.-L.Mon, 63(1994) 1-10, Evaluating weapon system by Analitical Hierarchy Process based on fuzzy scales, Fuzzy Sets and Systems.

11) T.L.Saaty, 1980, The analytical Hierarchy Process McGraw Hill, New York.

12) Bin Zhu, Zhan Wang, Haicheng Yang, Rong Mo and Yanwei Zhao, 2008. Applying fuzzy multiple attributes decision making for product configuration, Journal of Intelligent Manufacturing, 19, 591-598.

13) Xu, Z. S., 2002, Study on method for triangular fuzzy number based multi attribute decision making with preference information on alternatives. Systems Engineering and Electronics, 124 (18), 9-12.

14) Saaty, T.L., 1977, A Scaling Method for Priorities in Hierarchical Structures, J. Math. Psychology, 15, 234-281.

15) Yager, R.R., 1977, Multiple Objective Decision-Making Using Fuzzy Sets, Intl. J. Man-Machine Studies, 9, 375-382. 
International Journal on Soft Computing, Artificial Intelligence and Applications (IJSCAI), Vol.2, No.1, Feb 2013

16) Metin Dagdeviren, 2008, Decision making in equipment selection: an integrated approach with AHP and PROMETHEE, Journal of Intelligent Manufacturing, 19: 397-406.

17) Alim A. Fatah, Richard D. Arcilesi, James C. Peterson, Charlotte H. Lattin, Corrie Y. Wells and Joseph A. McClintock, 2007, "Guide for the Selection of Chemical Detection Equipment for Emergency First Responders", 3rd Edition, Guide 100-06, National Institute of Standards and Technology, Office of Law Enforcement Standards, January. www.eeel.nist.gov/oles/Publications/dhs100-06.pdf

18) Tricia Derringer, Thomas Kelly, Peter Bujnak, Robert Krile, Zachary Willenberg, Eric Koglin, 2006, "S-CAD Chemical Agent Detection System", Technology Evaluation Report, National Homeland Security Research Center, June. www.epa.gov/nhsrc/pubs/600r06140.pdf 